\title{
Magneto-optics of quasirelativistic electrons in graphene with an inplane electric field and in tilted Dirac cones in $\alpha$-(BEDT TTF $)_{2} \mathbf{I}_{3}$
}

\author{
Judit Sári, ${ }^{1,2}$ Mark O. Goerbig, ${ }^{3}$ and Csaba Tóke ${ }^{2}$ \\ ${ }^{1}$ Institute of Physics, University of Pécs, H-7624 Pécs, Hungary \\ ${ }^{2}$ BME-MTA Exotic Quantum Phases "Lendület” Research Group, Budapest University of Technology and Economics, \\ Institute of Physics, Budafoki út 8, H-1111 Budapest, Hungary \\ ${ }^{3}$ Laboratoire de Physique des Solides, CNRS UMR 8502, Université Paris-Sud, F-91405 Orsay Cedex, France
}

(Received 24 February 2015; revised manuscript received 1 July 2015; published 23 July 2015)

\begin{abstract}
Massless Dirac fermions occur as low-energy modes in several quasi-two-dimensional condensed matter systems such as graphene, the surface of bulk topological insulators, and in layered organic semiconductors. When the rotational symmetry in such systems is reduced either by an in-plane electric field or an intrinsic tilt of the Dirac cones, the allowed dipolar optical transitions evolve from a few selected transitions into a wide fan of interband transitions. We show that the Lorentz covariance of the low-energy carriers allows for a concise analysis of the emerging magneto-optical properties. We predict that infrared absorption spectra yield quantitative information on the tilted Dirac cone structure in organic compounds such as $\alpha$-(BEDT-TTF) ${ }_{2} \mathrm{I}_{3}$.
\end{abstract}

DOI: 10.1103/PhysRevB.92.035306

PACS number(s): 73.22.Pr, 73.61.Ph, 78.20.Ls

\section{INTRODUCTION}

The low-energy carriers in graphene imitate the relativistic physics of massless Dirac fermions with the Fermi velocity $v \approx c / 300$ replacing the speed of light $c$ [1]. Their peculiar Landau level (LL) structure provided the first evidence for the successful fabrication of monolayer graphene [2]. Experimental progress in the last decade has allowed, for example, the direct measurement of the Dirac cones in photoemission experiments [3], the observation of LLs in magneto-optical [4] and scanning-tunneling experiments [5], and the detection of Klein tunneling [6]. Further predictions follow from the Lorentz covariance of low-energy electrons in graphene, notably the collapse of Landau levels subjected to in-plane electric and perpendicular magnetic fields [7]. Here, conventional Landau states exist only as long as a Lorentz boost is possible to a reference frame where the external electric field vanishes; both the spectrum and the eigenstates follow by Lorentz covariance. Similar ideas apply to massless Dirac surfaces states of bulk topological insulators [8]. On the theory side, exploiting the Lorentz covariance of the massless Dirac equation often yields very concise derivations (compare, e.g., Refs. [7] and [19]), with the additional advantage of a unified understanding of a class of high-energy and low-energy phanomena.

More recently, the quasi-2D organic compound $\alpha$-(BEDT$\mathrm{TTF})_{2} \mathrm{I}_{3}$ has been shown to host Dirac cones at low energies [10,11] under pressure [12] or uniaxial strain [13]. Other quasi-2D organic materials that may share the same property, e.g., $\theta$-(BEDT-TTF) $)_{2} \mathrm{I}_{3}[14,15]$ and $\alpha$-(BEDT$\mathrm{TTF})_{2} \mathrm{NH}_{4} \mathrm{Hg}(\mathrm{SCN})_{4}$ [16], still need further investigation. In contrast to graphene, the Dirac cones in these materials are tilted (see Fig. 1). We will refer to such compounds as $2 D$ Weyl materials. In a magnetic field, this tilt plays the role of an effective in-plane electric field [17] and can be treated, as we show here, in a covariant manner. Moreover, the covariance of Dirac electrons in graphene and in $\alpha$-(BEDT-TTF $)_{2} \mathrm{I}_{3}$ yields another relativistic effect that consists of highly unusual magneto-optical transitions. Whereas in the frame of reference, where the electric field (or the tilt in 2D Weyl materials) vanishes, one obtains the usual dipolar transitions between the LLs $\pm n$ and $\pm(n \pm 1)$ [18], we predict that a Lorentz boost back to the laboratory frame gives rise to an amazing multiplication of these optical lines into a fan of experimentally observable interband transitions. Finally, we show that magneto-optical measurements may yield information on the tilt of the Dirac cones in 2D Weyl materials.

This article is structured as follows. In Sec. II, we review the low-energy band structure of the materials we study together with the relevant single-particle (Landau) states. In Sec. III, we study the matrix elements of the interaction of the electrons with electromagnetic radiation. The optical absorption per layer is analyzed in Sec. IV, where we also discuss how information can be gathered on the tilted Dirac cone structure of 2D Weyl materials from magneto-optics. In Sec. V, we conclude and discuss the experimental connections. Details of the calculation are delegated to the appendices.

\section{MATERIALS AND MODELS}

Let us first discuss graphene electrons in a perpendicular magnetic field $\mathbf{B}=B_{\perp} \mathbf{e}_{z}$ and in-plane electric field $\mathbf{E}=-E_{\|} \mathbf{e}_{y}$. The low-energy carriers are described by the Hamiltonian [1]

$$
\hat{H}_{\mathrm{G}}=v(\mathbf{p}+e \mathbf{A}) \cdot \boldsymbol{\sigma}-e E_{\|} y \mathbb{1},
$$

where $v$ is the Fermi velocity of graphene, the Pauli matrices are denoted by $\sigma_{i}$ with $i \in\{x, y, z\}$ and $\sigma=\left(\sigma_{x}, \sigma_{y}\right)$. Hereafter, we use the Landau gauge $\mathbf{A}=-y B_{\perp} \mathbf{e}_{x}$ and $e>0$. With the choice of $x^{\mu}=(v t, x, y)$ in the laboratory frame $\mathcal{R}$ one may introduce the 2D massless Dirac equation

$$
i \hbar v \gamma_{\mu}\left(\partial^{\mu}-\frac{i e}{\hbar} A^{\mu}\right) \Psi=0,
$$

where the representation $\gamma_{0}=\sigma_{z}, \gamma_{1}=-\sigma_{z} \sigma_{x}, \gamma_{2}=-\sigma_{z} \sigma_{y}$ has been used, and $A^{\mu}=\left(E_{\|} y / v,-y B_{\perp}, 0\right)$. The electric field can be eliminated by a Lorentz boost in the $x$ direction from the laboratory frame $\mathcal{R}$ to $\left(v t^{\prime}, x^{\prime}, y^{\prime}\right)=\Lambda(v t, x, y)=\left(\gamma\left(v_{D} t+\right.\right.$ $\left.\beta x), \gamma\left(v_{D} t+x\right), y\right)$ to another reference frame $\mathcal{R}^{\prime}$, as long as the drift velocity $v_{D}=E_{\|} / B_{\perp}$ is smaller than the Fermi 

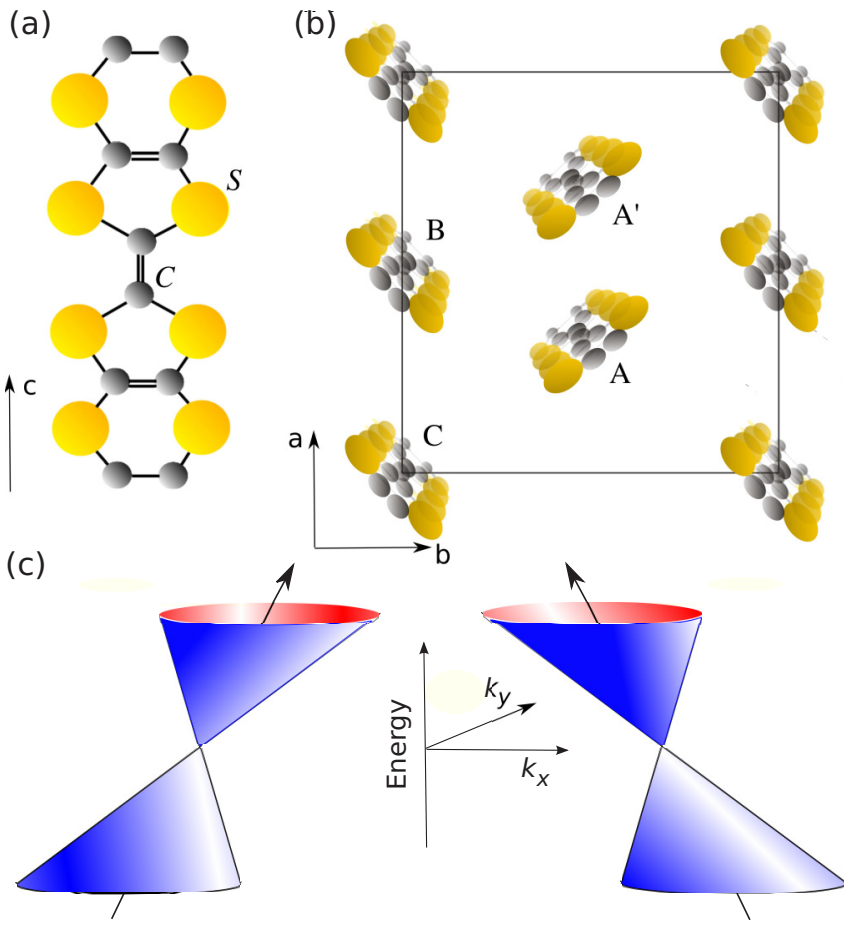

FIG. 1. (Color online) Organic compound $\alpha$-(BEDT-TTF) $)_{2} \mathrm{I}_{3}$. (a) Molecule (BEDT-TTF) that consists of carbon (C) and sulfur (S) atoms. (b) Arrangement of the four molecules (A, A', B, and C) in the unit cell of a single layer ( $a b$ plane). (c) Sketch of the low-energy band structure in the vicinity of the Fermi level that has the form of two tilted Dirac cones. We set up the coordinate system so that the cones are tilted in the $k_{x}$ direction.

velocity, $v_{D}<v$. The Lorentz boost is thus parametrized by the rapidity $\tanh \theta \equiv \beta=v_{D} / v$ and the Lorentz factor $\gamma=1 / \sqrt{1-\beta^{2}}$. The Hamiltonian in $\mathcal{R}^{\prime}$ then reads

$$
\hat{H}_{\mathrm{G}}^{\prime}=v\left(\mathbf{p}^{\prime}+e \mathbf{A}^{\prime}\right) \cdot \boldsymbol{\sigma},
$$

and covariance requires the two-spinor $\Psi$ to transform as

$$
\Psi^{\prime}\left(v t^{\prime}, x^{\prime}, y^{\prime}\right)=S(\Lambda) \Psi(v t, x, y),
$$

with $S(\Lambda)=\exp \left(\frac{\theta}{2} \sigma_{x}\right)$.

Notice that the simple form of $\hat{H}_{\mathrm{G}}^{\prime}$ allows for a straightforward diagonalization in $\mathcal{R}^{\prime}$ by standard techniques $[7,9,19]$. The sought eigenstates of $\hat{H}_{\mathrm{G}}$ are then obtained by a transformation back to $\mathcal{R}$

$$
\begin{aligned}
\Psi_{\lambda, n ; k}(x, y)= & \frac{1}{\sqrt{2}}\left[\left(\begin{array}{c}
\sinh \frac{\theta}{2} \\
-\cosh \frac{\theta}{2}
\end{array}\right) \phi_{\lambda, n ; k}(x, y)\right. \\
& \left.+\lambda\left(\begin{array}{c}
\cosh \frac{\theta}{2} \\
-\sinh \frac{\theta}{2}
\end{array}\right) \phi_{\lambda, n-1 ; k}(x, y)\right]
\end{aligned}
$$

for $n \geqslant 1$ and

$$
\Psi_{n=0 ; k}(x, y)=\left(\begin{array}{c}
\sinh \frac{\theta}{2} \\
-\cosh \frac{\theta}{2}
\end{array}\right) \phi_{n=0 ; k}(x, y)
$$

for $n=0$, where $\lambda= \pm$ denotes LLs of positive and negative energy, respectively, and $k$ is the wave vector in the $x$ direction related to the conserved momentum component $p_{x}=\hbar k$. Here, we have used the functions

$$
\begin{gathered}
\phi_{\lambda, n ; k}(x, y)=\frac{e^{i k x}}{\sqrt{2 \pi}} \frac{1}{\sqrt{\sqrt{\pi \gamma} \ell 2^{n} n !}} H_{n}\left(\eta_{\lambda, n}\right) e^{-\eta_{\lambda, n}^{2} / 2}, \\
\eta_{\lambda, n}=\frac{1}{\sqrt{\gamma} \ell}\left(y-k \ell^{2}\right)-\lambda \beta \sqrt{2 n},
\end{gathered}
$$

where $\ell=\sqrt{\hbar / e B}$ is the magnetic length, $H_{n}$ is a Hermite polynomial, and $\delta$-normalization has been used for the wave functions. The covariant character is apparent here in the argument $\eta_{\lambda, n}$ of the wave functions, where one notices the contraction factor $1 / \sqrt{\gamma}$. The second term reflects the admixing of the timelike component, $\lambda \sqrt{2 n}$, proportional to the LLs,

$$
\epsilon_{\lambda, n ; k}^{\mathrm{G}}=\frac{\lambda \hbar v}{\gamma^{3 / 2} \ell} \sqrt{2 n}-\hbar v_{D} k,
$$

which exhibit a collapse in the $v_{D} \rightarrow v(\gamma \rightarrow \infty)$ limit [7,19]. The last term in Eq. (8) stands for the dependence of the single-particle energies on the location of the guiding center if an in-plane electric field is present. This term is also present in the case of nonrelativistic electrons, but the LL spacing of the latter is insensitive to the inplane electric field, in contrast to the decreased LL spacing of relativistic electrons apparent in the factor $1 / \gamma^{3 / 2}$ in Eq. (8).

The low-energy carriers in 2D Weyl materials are described by the minimal Weyl Hamiltonian $[11,20,21]$, which allows for different velocities $v_{x}, v_{y}$ in two directions, and a shift of the centers of the equienergy ellipses in a generic tilt direction. The anisotropy $v_{x} \neq v_{y}$ can be eliminated by rescaling $y^{\prime}=\left(v_{x} / v_{y}\right) y$, and rotating the direction of the tilt into the $x$ direction. The details of this procedure are presented in Appendix A. We obtain

$$
\hat{H}_{\mathrm{W}}=v_{x}(\mathbf{p}+e \mathbf{A}) \cdot \boldsymbol{\sigma}+v_{0}\left(p_{x}+e A_{x}\right) \mathbb{1} .
$$

Here, $v_{0}$ is a velocity parameter that is related to the band structure, c.f. Eq. (A7).

In the Landau gauge the noncovariant term $v_{0} p_{x} \mathbb{1}$ in Eq. (9) is harmless as $p_{x}$ only extracts a quantum number. The Hamiltonian (9) can be rewritten in the form $\hat{H}_{\mathrm{W}}=$ $\hat{H}_{\mathrm{W}}^{\text {cov. }}+v_{0} p_{x} \mathbb{1}$, where the last term is diagonal both with respect to the sublattice $\sigma$ and in the wave vector $k$. The energy levels can therefore be written as

$$
\epsilon_{\lambda, n ; k}^{\mathrm{W}}=\epsilon_{\lambda, n ; k}^{\text {cov. }}+\hbar v_{0} k,
$$

where $\epsilon_{\lambda, n ; k}^{\text {cov. }}$ are the solutions of the Hamiltonian's covariant part $\hat{H}_{\mathrm{W}}^{\text {cov. }}$, which has the same form as that in Eq. (1). In the Landau gauge, the term $e v_{0} A_{x} \mathbb{1}=-e v_{0} B_{\perp} y \mathbb{1}$ can be viewed as generated by an effective pseudoelectric field $E_{\|}^{\text {eff }}=B_{\perp} v_{0}$, and $v_{0}$ assumes the role of the drift velocity. The wave functions are obtained by methods that are completely analogous to the graphene case, using $\tanh \theta \equiv \beta=v_{0} / v$ in the Lorentz boost. Then the LL spectrum becomes [20,21]

$$
\epsilon_{\lambda, n ; k}^{\mathrm{W}}=\frac{\lambda \hbar v}{\gamma^{3 / 2} \ell} \sqrt{2 n},
$$

where an average velocity $v=\sqrt{v_{x} v_{y}}$ has been introduced. Comparing with Eq. (8), the guiding-center-dependent term is absent, because there is no inplane electric field. 
Both in graphene and in 2D Weyl materials, Dirac fermions occur in two cones related by time-reversal symmetry. Whereas electrons in both valleys are subjected to the same external electric field (drift velocity), the tilt in Weyl materials is opposite in the two valleys and so is the implied effective electric field.

\section{INTERACTION WITH ELECTROMAGNETIC RADIATION}

Let the 2D sample be located in the $z=0$ plane. We consider the interaction with a classical radiation field

$$
\mathbf{A}^{\mathrm{rad}}(\mathbf{r}, t)=\operatorname{Re}\left[\frac{E_{0}}{\omega}\left(\alpha \hat{\mathbf{e}}_{x}+\tau \hat{\mathbf{e}}_{y}\right) e^{i \omega t}\right],
$$

where $|\alpha|^{2}+|\tau|^{2}=1$ and $\omega$ is fixed, $\mathbf{A}^{\mathrm{rad}}(\mathbf{r}, t)$ is added to the vector potential in Eqs. (1) and (9). Notice that the different single-particle Hamiltonians for graphene and 2D Weyl materials yield slightly different perturbation operators

$$
{ }^{\mathrm{G}} \delta \hat{H}=e v \frac{E_{0}}{\omega}\left(\alpha \sigma_{x}+\tau \sigma_{y}\right)
$$

and

$$
{ }^{\mathrm{w}} \delta \hat{H}=e v_{x} \frac{E_{0}}{\omega}\left(\tilde{\alpha} \sigma_{x}+\tilde{\tau} \sigma_{y}+\frac{v_{0}}{v_{x}} \tilde{\alpha} \mathbb{1}\right),
$$

respectively. The parameters $\alpha$ and $\tau$ determine the light polarization, e.g., $\alpha=1 / \sqrt{2}$ and $\tau=\mp i / \sqrt{2}$ for circular polarization $\circlearrowright / \circlearrowleft$. In the case of 2D Weyl materials, $\alpha$ and $\tau$ are also affected by the rescaling of the coordinates (c.f. Appendix A); the use of tildes for $\tilde{\alpha}$ and $\tilde{\tau}$ refer to this change.

For the electron-light interaction, we will need the matrix elements of $\delta \hat{H}^{\mathrm{G} / \mathrm{W}}$ :

$$
\mathrm{G} / \mathrm{W} \delta H_{\lambda, n, k}^{\lambda^{\prime}, n^{\prime}, k^{\prime}}=e v \frac{E_{0}}{\omega} \delta\left(k-k^{\prime}\right)\left({ }^{\mathrm{G} / \mathrm{W}} Q_{\lambda, n}^{\lambda^{\prime}, n^{\prime}}\right),
$$

where the dimensionless factors ${ }^{\mathrm{G}} Q_{\lambda, n}^{\lambda^{\prime}, n^{\prime}}$ and ${ }^{\mathrm{W}} Q_{\lambda, n}^{\lambda^{\prime}, n^{\prime}}$ of these matrix elements are

$$
\begin{aligned}
{ }^{\mathrm{G}} Q_{\lambda, n}^{\lambda^{\prime}, n^{\prime}}= & -\frac{1}{2}\left[F_{n, n^{\prime}}^{\lambda, n, \lambda^{\prime}, n^{\prime}} \alpha \beta+\lambda^{\prime} \lambda F_{n-1, n^{\prime}-1}^{\lambda, n, \lambda^{\prime}, n^{\prime}} \alpha \beta\right. \\
& \left.+\lambda^{\prime} F_{n, n^{\prime}-1}^{\lambda, n \lambda^{\prime}, n^{\prime}}\left(\alpha+i \tau \gamma^{-1}\right)+\lambda F_{n-1, n^{\prime}}^{\lambda, n, \lambda^{\prime}, n^{\prime}}\left(\alpha-i \tau \gamma^{-1}\right)\right]
\end{aligned}
$$

and

$$
\begin{aligned}
{ }^{\mathrm{W}} Q_{\lambda, n}^{\lambda^{\prime}, n^{\prime}}= & \frac{1}{2 \gamma}\left[\lambda^{\prime} F_{n, n^{\prime}-1}^{\lambda, n \lambda^{\prime}, n^{\prime}}\left(\gamma^{-1} \tilde{\alpha}-i \tilde{\tau}\right)\right. \\
& \left.+\lambda F_{n-1, n n^{\prime}}^{\lambda, n, \lambda^{\prime}, n^{\prime}}\left(\gamma^{-1} \tilde{\alpha}+i \tilde{\tau}\right)\right] .
\end{aligned}
$$

The functions $F_{q, r}^{\lambda, s, \lambda^{\prime}, p}$ are defined in terms of associated Laguerre polynomials $L_{n}^{m}$ as

$$
\begin{aligned}
F_{q, r}^{\lambda, s, \lambda^{\prime}, p} & =\sqrt{\frac{r !}{q !}} e^{-\left(z_{\lambda, s}^{\lambda^{\prime}, p}\right)^{2} / 2}\left(z_{\lambda, s}^{\lambda^{\prime}, p}\right)^{q-r} L_{r}^{q-r}\left[\left(z_{\lambda, s}^{\lambda^{\prime}, p}\right)^{2}\right], \\
z_{\lambda, s}^{\lambda^{\prime}, p} & =\beta\left(\lambda^{\prime} \sqrt{p}-\lambda \sqrt{s}\right),
\end{aligned}
$$

for $q \geqslant r$, otherwise $F_{q, r}^{\lambda, s, \lambda^{\prime}, p}=F_{r, q}^{\lambda^{\prime}, p, \lambda, s}$.

We show $\left|{ }^{\mathrm{G} / \mathrm{W}} Q_{\lambda, n}^{\lambda^{\prime}, n^{\prime}}\right|^{2}$ in Fig. 2 for graphene and 2D Weyl materials for $\beta=0.2$ and 0.8 , assuming right circular
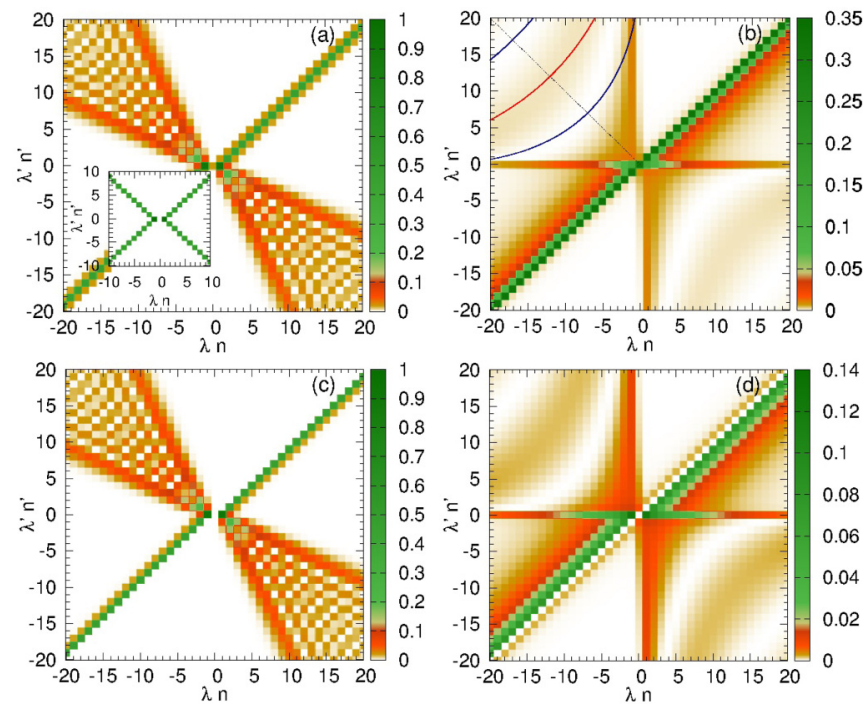

FIG. 2. (Color online) (a) and (b) The matrix elements $\left|{ }^{\mathrm{G}} Q_{\lambda, n}^{\lambda^{\prime}, n^{\prime}}\right|^{2}$ for graphene in crossed $B_{\perp}$ and $E_{\|}$fields, for right circularly polarized light. (c) and (d) $\left|{ }^{\mathrm{W}} Q_{\lambda, n}^{\lambda^{\prime}, n^{\prime}}\right|^{2}$ for a 2D Weyl material, for elliptical polarization that appears as right circularly polarized in the rotated and rescaled system. (a) and (c) $\beta=0.2$ and (b) and (d) $\beta=0.8$. Inset of (a): the same as in (a) for $E_{\|}=0$ limit. In (b), the dotted line indicates the matrix elements corresponding to $n^{\prime}=n \geqslant 1, \lambda^{\prime}=-\lambda$ transitions, see Eq. (19); blue (red) curves indicate equienergy lines with minimal (maximal) value of the matrix elements.

polarization and a special choice of elliptical polarization, respectively. The latter choice is determined by the asymmetric velocities and corresponds to a circular polarization after rescaling. The opposite polarization is obtained via the relation

$$
\left|\left(Q^{\circlearrowleft}\right)_{\lambda, n}^{\lambda^{\prime}, n^{\prime}}\right|^{2}=\left|\left(Q^{\circlearrowright}\right)_{\lambda, n^{\prime}}^{\lambda^{\prime}, n}\right|^{2},
$$

and both $\left|\left(Q^{\circlearrowleft /}\right)_{\lambda, n}^{\lambda^{\prime}, n^{\prime}}\right|^{2}$ and $\left|\left(Q^{\circlearrowright}\right)_{\lambda, n}^{\lambda^{\prime}, n^{\prime}}\right|^{2}$ are independent of the sign of $\beta$. The results for both systems are almost identical, apart from small differences due to the slightly different perturbation operators in Eqs. (12) and (13). Most saliently, we obtain novel transitions beyond the usual dipolar ones, $\propto \delta_{n^{\prime}, n \pm 1}$ that arise for vanishing $E_{\|}$or no tilt, respectively, and which apply therefore only in the reference frame $\mathcal{R}^{\prime}$, where rotation symmetry is restored. Alternatively, one may thus view the occurrence of additional transitions as due to the broken rotation symmetry caused by the Lorentz boost back to the laboratory frame $\mathcal{R}$. The weight of intraband transitions that change the LL index $n$ by more than one unit grows monotonically with $\beta$ whereas the interband transitions show a complex fan structure with an arc pattern. The fan, whose opening angle increases with $\beta$, is bounded by large values of the perturbation matrix element that are not affected by the arc pattern [c.f. also the inset of Fig. 4(b)].

For further insight into the origin of the observed arc pattern, consider the case $n^{\prime}=n \geqslant 1, \lambda^{\prime}=-\lambda$ for large $\beta$. One finds that

$$
{ }^{\mathrm{G} / \mathrm{w}} Q_{\lambda, n}^{-\lambda, n} \propto e^{-2 \beta^{2} n} L_{n-1}^{1}\left(4 \beta^{2} n\right),
$$

where the associated Laguerre polynomial $L_{n}^{m}$ can be generalized to continuous $n$ in terms of the confluent hypergeometric 


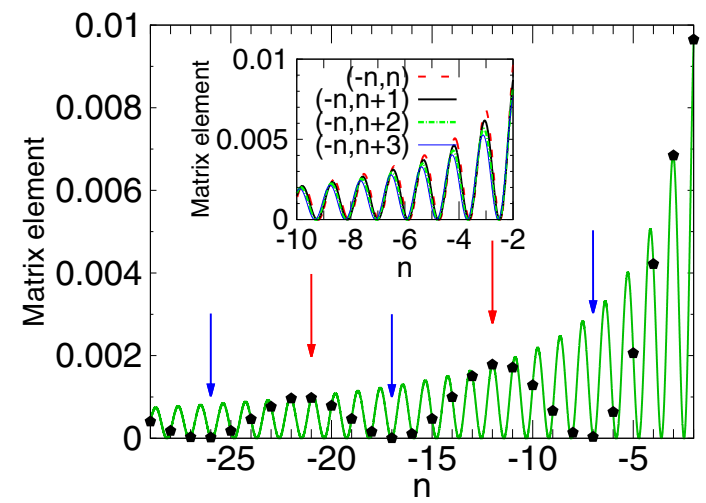

FIG. 3. (Color online) The matrix element $\left|{ }^{\mathrm{G}} Q_{\lambda, n}^{-\lambda, n}\right|^{2}$ as a continuous function of $n$ (green) for $\beta=0.8$. The black dots indicate integer values of $n$. (Inset) The same along the lines $\lambda^{\prime}=-\lambda$ and $n^{\prime}=n+c$, where $c=1,2,3$.

function. At fixed $\beta$, this function has decaying oscillations in $n$. For $\beta=0.8$, this function is plotted in Fig. 3(a), where we have used $n$ as a continuous variable. The apparent period $\delta n$ is more than, but close to, unity, and this incongruity with integer values yields a beating with a distance $\Delta n \approx 10$ between consecutive zeros that gives rise to the observed arc pattern. By Nyquist's sampling theorem, this effect survives as long as $\delta n<2$ (which corresponds to $\beta \gtrsim 0.4$ ). Nearby transitions such as $\left(\lambda^{\prime}, n^{\prime}\right)=(-\lambda, n+1),\left(\lambda^{\prime}, n^{\prime}\right)=(-\lambda, n+2)$, etc., can be analyzed similarly. Remarkably, their beatings are of almost identical period and are in phase (Fig. 3 inset). While the latter phase condition does not hold for small $\beta$ in general, we will see below that two characteristic periods can be extracted, one of which is continuously connected to the period at large $\beta$.

\section{MAGNETO-OPTICAL ABSORPTION}

By Fermi's golden rule [26], the transition rates per unit area $A$ are

$$
\begin{aligned}
R_{\lambda, n ; k \rightarrow \lambda^{\prime}, n^{\prime} ; k^{\prime}}^{\mathrm{emi} / \mathrm{abs}}= & \left.\left.\frac{1}{A} \frac{2 \pi}{\hbar}\right|^{\mathrm{G} / \mathrm{W}} \delta H_{\lambda, n, k}^{\lambda^{\prime}, n^{\prime}, k^{\prime}}\right|^{2} \\
& \times \delta\left(\epsilon_{\lambda^{\prime}, n^{\prime}}-\epsilon_{\lambda, n} \pm \hbar \omega\right) n_{F}\left(\epsilon_{\lambda, n}\right)\left[1-n_{F}\left(\epsilon_{\lambda^{\prime}, n^{\prime}}\right)\right],
\end{aligned}
$$

where $n_{F}(\epsilon)$ is the Fermi function. Integrating over all states, the total transition rate per unit area is

$$
R=\sum_{\lambda, n, \lambda^{\prime}, n^{\prime}} \int \mathrm{d} k \int \mathrm{d} k^{\prime}\left(R_{\lambda, n ; k \rightarrow \lambda^{\prime}, n^{\prime} ; k^{\prime}}^{\mathrm{abs}}-R_{\lambda^{\prime}, n^{\prime} ; k^{\prime} \rightarrow \lambda, n ; k}^{\mathrm{emi}}\right) .
$$

This can be normalized using the absorbed/emitted energies and the magnitude of the Poynting vector $S=E_{0}^{2} / 2 \mu_{0} c$,

$$
i_{\mathrm{abs}}^{\mathrm{G} / \mathrm{W}}(\omega)=\frac{R \hbar \omega}{S},
$$

which is dimensionless. (The celebrated $2.3 \%$ per sheet absorbtion of pristine graphene[28] employs this definition.) We introduce a Lorentzian broadening $\Gamma$ for neglected effects (temperature, impurities, phonons, etc). Taking spin and valley
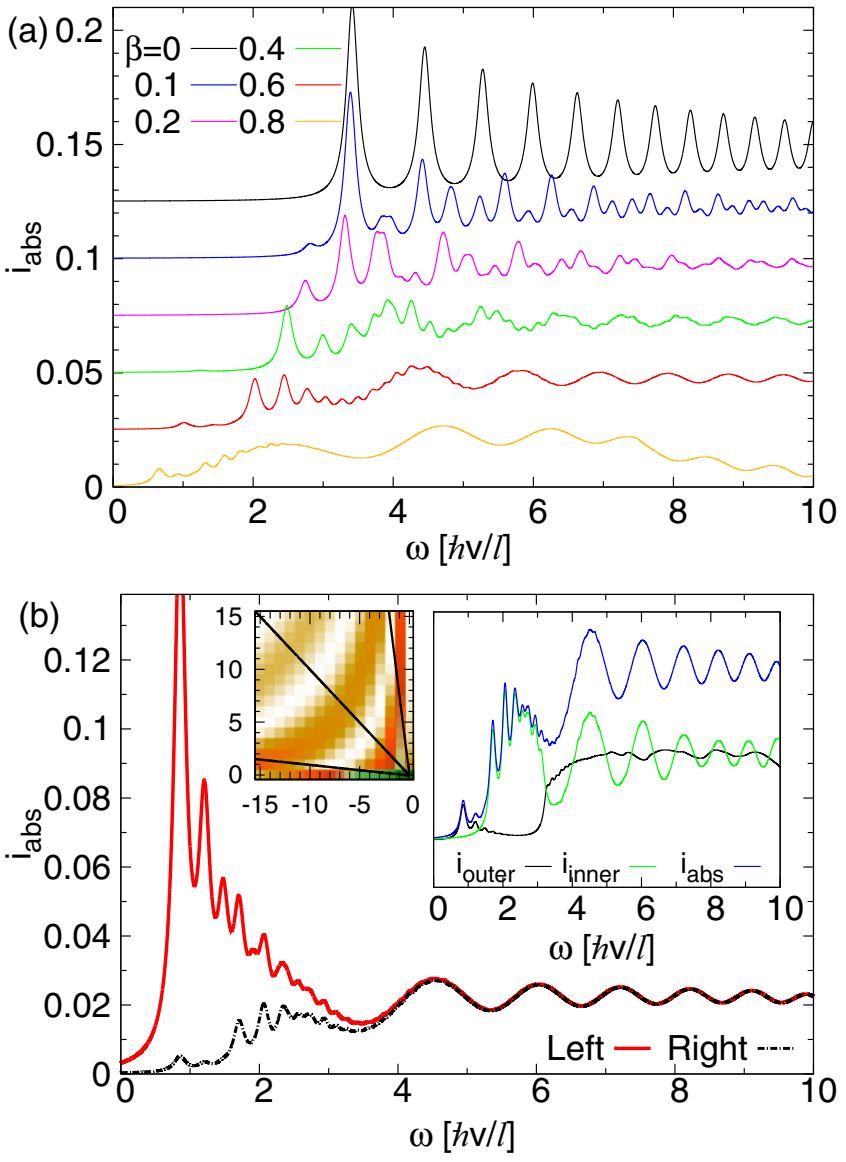

FIG. 4. (Color online) (a) Absorption coefficient of graphene in perpendicular magnetic and in-plane electric fields for right circularly polarized light. Curves are shifted for clarity. (b) Comparison of $i_{\text {abs }}$ for right and left circularly polarized light for $\beta=0.7$. Inset: The total $i_{\text {abs }}, i_{\text {inner }}$ due to the inner part of the interband fan, and $i_{\text {outer }}$ due to the outer part, for graphene $\beta=0.7$.

into account, one obtains

$$
\begin{aligned}
i_{\mathrm{abs}}^{\mathrm{G} / \mathrm{W}}(\omega)= & \frac{2 v^{2} \hbar \mu_{0} c e^{2}}{\ell^{2}} \sum_{\lambda, n, \lambda^{\prime}, n^{\prime}} \frac{n_{\mathrm{F}}\left(\epsilon_{\lambda, n}\right)-n_{\mathrm{F}}\left(\epsilon_{\lambda^{\prime}, n^{\prime}}\right)}{\epsilon_{\lambda^{\prime}, n^{\prime}}-\epsilon_{\lambda, n}} \\
& \times\left.\left.\right|^{\mathrm{G} / \mathrm{W}} Q_{\lambda, n}^{\lambda^{\prime}, n^{\prime}}\right|^{2} \frac{1}{\pi} \frac{\Gamma}{\Gamma^{2}+\left(\epsilon_{\lambda^{\prime}, n^{\prime}}-\epsilon_{\lambda, n}-\hbar \omega\right)^{2}} .
\end{aligned}
$$

Inspecting the graphene data by Sadowski et al. [4], we will use $\Gamma=0.1 \hbar v / \ell$ as a rough estimation. Equation (18) implies that the absorption for opposite polarizations are connected

$$
i_{\mathrm{abs}}^{\mathrm{G} / \mathrm{W}, \circlearrowleft}(\omega, \mu)=i_{\mathrm{abs}}^{\mathrm{G} / \mathrm{W}, \circlearrowright}(\omega,-\mu),
$$

where $\mu$ is the chemical potential.

The absorption of graphene in perpendicular magnetic and in-plane electric field is plotted as a function of frequency in Fig. 4(a) for several values of $\beta$. Whereas for low frequencies, one finds well-pronounced lines corresponding to individual transitions, in the large frequency domain the lines of many allowed transitions coalesce. The resulting supermodulation stems from the arc pattern in Fig. 2, where the arcs connect different transitions of nearby energies, see the lines 

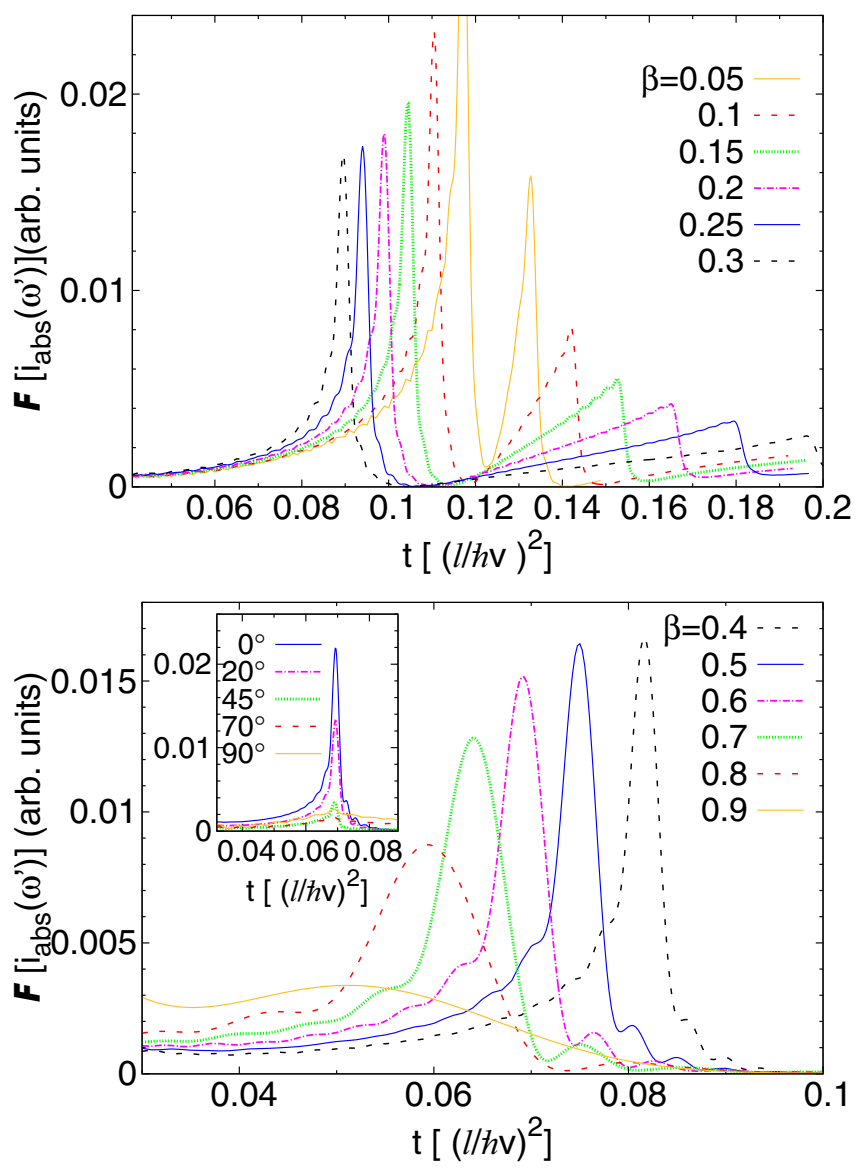

FIG. 5. (Color online) (a) The one-sided Fourier-transformed rescaled absorption spectrum $\mathcal{F}\left[i_{\text {abs }}\left(\omega^{\prime}\right)\right](t)$ for linearly polarized light exhibits two characteristic peaks in the range $\beta \leqslant 0.3$. We show the case of $2 \mathrm{D}$ Weyl materials; the graphene case is similar. (b) For $\beta \geqslant 0.4$, only a single characteristic peak is discernible. (Inset) The dependence of the characteristic peak on the direction of linear polarization at $\beta=0.6$, relative to the tilt direction.

connecting minima/maxima in Fig. 2(b) for illustration. In Fig. 4(b), we compare the absorption for the two circular light polarizations. The low-frequency absorption, where only few LL transitions contribute, depends strongly on polarization. In contrast, the absorption becomes polarization-independent at high frequencies because of the coalescence of a large number of transitions. However, the effect of the above-mentioned supermodulation is retained. Finally, in the inset of Fig. 4(b), we separate the contributions of the patterned inner wedge of the fan of interband transitions and of the bounding lines to $i_{\text {abs }}(\omega)$; we find that the latter merely yields a constant background.

All features in the optical spectra follow the $\epsilon_{\lambda, n ; k}^{\mathrm{G} / \mathrm{W}} \propto \sqrt{n}$ scaling of massless 2D Dirac fermions. Therefore we consider the spectra as a function of $\omega^{\prime}=\omega^{2}$, and seek the effect of the high-frequency regular pattern in their one-sided Fourier transform (or Laplace transform with an imaginary argument),

$$
\mathcal{F}\left[i_{\text {abs }}\left(\omega^{\prime}\right)\right](t)=\int_{0}^{\infty} \mathrm{d} \omega^{\prime} e^{-i 2 \pi t \omega^{\prime}} i_{\text {abs }}\left(\omega=\sqrt{\omega^{\prime}}\right) .
$$

A characteristic peak emerges, as shown in the inset of Fig. 5 for 2D Weyl materials, even for linearly polarized
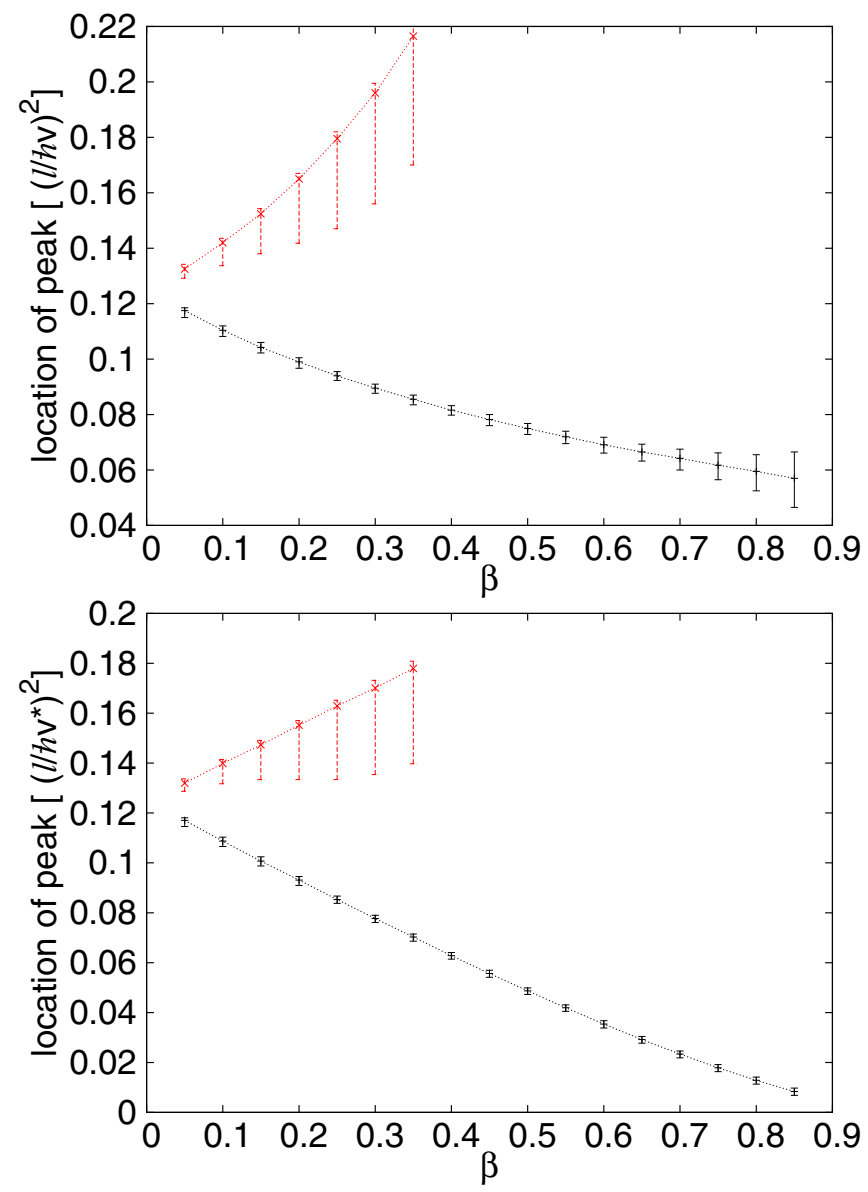

FIG. 6. (Color online) (a) The location of the main peak(s) in the one-sided Fourier-transform of the rescaled absorption spectrum $\mathcal{F}\left[i_{\text {abs }}\left(\omega^{\prime}\right)\right](t)$ for linearly polarized light as a function of the tilt parameter $\beta$ for a 2D Weyl material. Error bars indicate the FWHM assuming a Lorentzian broadening. (b) The same data plotted in units of $\left(\ell / \hbar v^{*}\right)^{2}$, where the angle-averaged Fermi velocity $v^{*}=$ $\sqrt{v_{x} v_{y}}\left(1-\beta^{2}\right)^{3 / 4}$ is directly related to the density of states that appears in several experiments.

light. A particular polarization direction exists for which the visibility is optimal. For graphene, this is the direction of the electric field; for 2D Weyl materials, it is the tilt direction. For small $\beta \lesssim 0.35$, a satellite peak appears [c.f. Fig. 5(a) for the case of 2D Weyl materials], but the main peak remains clearly visible.

Figure 6 shows the relation between the tilt parameter $\beta$ and the location of the two peaks in the one-sided Fourier transform of the rescaled spectrum in Eq. (25). Here, we restrict our attention to 2D Weyl materials. Extracting $\beta$ is straightforward in the moderate-tilt limit, $\beta \lesssim 0.35$, via the splitting of the two peaks in $\mathcal{F}\left[i_{\text {abs }}\left(\omega^{\prime}\right)\right]$. In the large-tilt region $\beta \gtrsim 0.35$, however, there is only one peak. Its location is informative, but for quantitative analysis we also need to know the Fermi velocity. Then it is more useful to plot the location of the peak in units of $\left(\ell / \hbar v^{*}\right)^{2}$, where the effective Fermi velocity $v^{*}=$ $\sqrt{v_{x} v_{y}} / \gamma^{3 / 2}=\sqrt{v_{x} v_{y}}\left(1-\beta^{2}\right)^{3 / 4}$ takes into account both the anisotropy and the relativistic reduction of the energy scale. This velocity $v^{*}$ occurs directly in the density of states [20,21], or in the LL spacing in Eq. (10). Thus we can obtain the tilt 
parameter only if we utilize independent information on $v^{*}$ from thermodynamics or low-frequency magneto-optics.

\section{CONCLUSION}

In conclusion, the pseudorelativistic nature of electrons in graphene in crossed electric and magnetic fields, and in 2D Weyl materials such as $\alpha$-(BEDT-TTF $)_{2} \mathrm{I}_{3}$ manifests itself in unique magneto-optical properties. A large number of transitions beyond the usual dipolar ones become possible. Their coalescence at high frequencies is the fingerprint of the broken rotation invariance in both cases either due to the particular tilt direction or that of the electric field.

In graphene, this effect might be observed easier than the earlier predicted Landau level collapse [7], because the magneto-optical effect is present in the low- $\beta$ range where the change of the Landau level energies is still small. A side-gated geometry must be necessary. Here, however, the screening of the external electric field by the edge states $[22,23]$ might be a complication [24]. We note that an upper limit to the achievable $\beta$ values in graphene is set by the condition that potential energy change over a magnetic length should be less then the energy difference between adjecent LLs to avoid tunneling along the parallel electric field. For the $n=0,1$ Landau levels, this means $e E_{\|} \ell<\epsilon_{1}^{\mathrm{G}}-\epsilon_{0}^{\mathrm{G}}=\frac{\hbar v \sqrt{2}}{\ell}\left(1-\beta^{2}\right)^{3 / 4}$, which sets $\beta<0.75$.

In $\alpha$-(BEDT-TTF $)_{2} \mathrm{I}_{3}$ and potentially other quasi-2D organic materials with massless Dirac cones [14-16], on the other hand, a quantitative analysis of this fingerprint, in combination with other information on Fermi velocities if the tilt turns out to be large, helps us determine the tilting parameters of the massless Dirac cones. We notice finally that the same magneto-optical features should appear in strained graphene, where uniaxial strain yields tilted Dirac cones [20] that are predicted to show a clear signature also in Raman spectroscopy [25].

\section{ACKNOWLEDGMENTS}

We acknowledge support from the Hungarian Scientific Research Funds No. K105149. C.T. was supported by the Hungarian Academy of Sciences. Supercomputer facilities were provided by National Information Infrastructure Development Institute, Hungary. J.S. was partially supported by Campus France supervised by the French Ministry of Education, and also acknowledges hospitality from Laboratoire de Physique des Solides of Université Paris-Sud. We thank M. Monteverde, C. Faugeras, M. Orlita, M. Potemski, A. Pályi, and K. Kamarás for useful discussions.

\section{APPENDIX A: DERIVATION OF THE MINIMAL WEYL HAMILTONIAN}

Massless Dirac carriers of $\alpha$-(BEDT-TTF $)_{2} \mathrm{I}_{3}$ are described by the minimal Weyl Hamiltonian [11] using four parameters $v_{0}^{x}, v_{0}^{y}, v_{x}, v_{y}$ as follows:

$$
\hat{H}_{\mathrm{W}}=v_{x} \hat{p}_{x} \sigma_{x}+v_{y} \hat{p}_{y} \sigma_{y}+\left(v_{0}^{x} \hat{p}_{x}+v_{0}^{y} \hat{p}_{y}\right) \mathbb{1} .
$$

The inclination of the Dirac cone is determined by the combined effect of the tilt and the anisotropy. We characterize the anisotropy of the Dirac cones by the quotient $v_{x} / v_{y}$. Even if $v_{x}=v_{y}$, the constant energy slices are not concentric because $\left(v_{0}^{x}, v_{0}^{y}\right) \neq(0,0)$. We quantify this tilt by the parameter

$$
\eta=\sqrt{\left(v_{0}^{x} / v_{x}\right)^{2}+\left(v_{0}^{y} / v_{y}\right)^{2}} .
$$

Following Refs. [21] and [27], we use a rescaled and rotated coordinate system, defined by the transformation

$$
\begin{aligned}
\left(\begin{array}{c}
\tilde{x} \\
\tilde{y}
\end{array}\right) & =\left(\begin{array}{cc}
\cos \phi & \sin \phi \\
-\sin \phi & \cos \phi
\end{array}\right)\left(\begin{array}{c}
x \\
y \frac{v_{x}}{v_{y}}
\end{array}\right), \\
\left(\begin{array}{c}
\tilde{p}_{x} \\
\tilde{p}_{y}
\end{array}\right) & =\left(\begin{array}{cc}
\cos \phi & \sin \phi \\
-\sin \phi & \cos \phi
\end{array}\right)\left(\begin{array}{c}
\hat{p}_{x} \\
\hat{p}_{y} \frac{v_{y}}{v_{x}}
\end{array}\right),
\end{aligned}
$$

in terms of the rotation angle $\phi$. Rescaling $\hat{p}_{y}$ removes the anisotropy, and the rotation brings $\tilde{p}_{x}$ in the tilt direction if we choose

$$
\cos \phi=\frac{v_{0}^{x} v_{y}}{\sqrt{\left(v_{0}^{y} v_{x}\right)^{2}+\left(v_{0}^{x} v_{y}\right)^{2}}}
$$

Then the four-parameter Hamiltonian $\hat{H}_{\mathrm{W}}$ can be rewritten in a simpler form

$$
\hat{H}_{\mathrm{W}}=v_{x} \tilde{\mathbf{p}} \cdot \boldsymbol{\sigma}+v_{0} \tilde{p}_{x} \mathbb{1},
$$

where $\tilde{\mathbf{p}}$ is measured from the direction of the tilt, and

$$
v_{0}=\eta v_{x}
$$

The Peierls substitution in Eq. (A6) then yields Eq. (9).

\section{APPENDIX B: DETERMINATION OF THE CUTOFF}

As the number of Landau levels within the range of validity of the Weyl Hamiltonian is limited, we examine the robustness of our results against the change of the Landau level cutoff.

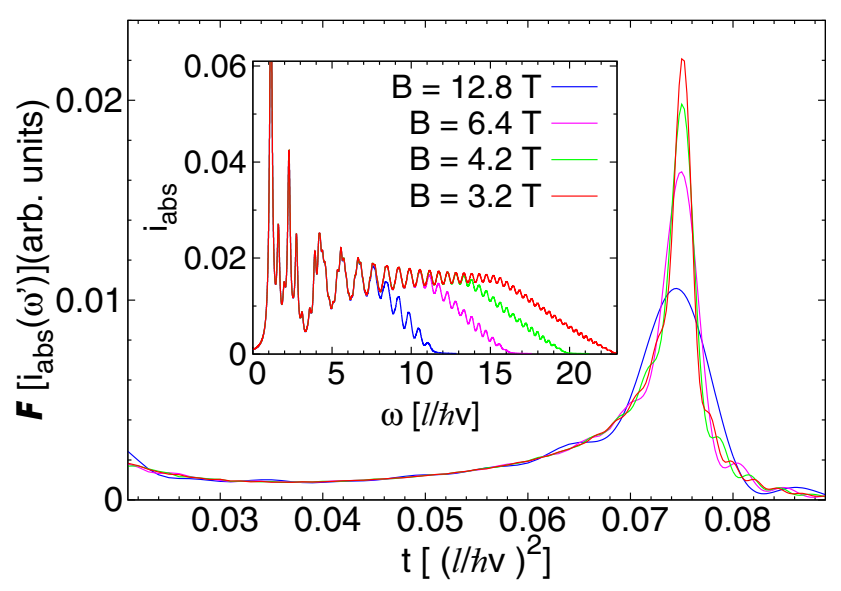

FIG. 7. (Color online) The one-sided Fourier transform of $i_{\mathrm{abs}}^{\mathrm{W}}(\omega)$ for the Weyl Hamiltonian for linearly polarized light in the tilt direction [Eq. (A5)] and $\beta=0.5$ for various Landau level cutoff values: $25,50,100,150$, which corresponds to $B=12.8,6.4,4.2,3.2$ $\mathrm{T}$, respectively. (Inset) The original $i_{\mathrm{abs}}^{\mathrm{W}}(\omega)$ function. 
Previously, we estimated the number of available Landau levels is $300 / B_{\perp}$ [27]. Figure 7 shows the effect of an arbitrary change of the cutoff. The oscillatory behavior remains visible in the $i_{\text {abs }}^{\mathrm{W}}(\omega)$ curves even at low cutoffs. The relevant peaks of $\mathcal{F}\left[i_{\text {abs }}\left(\omega^{\prime}\right)\right]$ also survive, though with reduced height. We can see that the oscillatory behavior of $i_{\mathrm{abs}}^{\mathrm{W}}$ is present for as few as 50 Landau levels, which is a safe choice for reasonable magnetic fields.
[1] P. R. Wallace, Phys. Rev. 71, 622 (1947); N. H. Shon and T. Ando, J. Phys. Soc. Jpn. 67, 2421 (1998); G. W. Semenoff, Phys. Rev. Lett. 53, 2449 (1984).

[2] K. S. Novoselov, A. K. Geim, S. V. Morozov, D. Jiang, M. I. Katsnelson, I. V. Grigorieva, S. V. Dubonos, and A. A. Firsov, Nature (London) 438, 197 (2005); Y. Zhang, Y.-W. Tan, H. L. Stormer, and P. Kim, ibid. 438, 201 (2005).

[3] For a review, see D. N. Basov, M. M. Fogler, A. Lanzara, F. Wang, and Y. Zhang, Rev. Mod. Phys. 86, 959 (2014).

[4] M. L. Sadowski, G. Martinez, M. Potemski, C. Berger, and W. A. de Heer, Phys. Rev. Lett. 97, 266405 (2006).

[5] G. Li, A. Luican, and E. Y. Andrei, Phys. Rev. Lett. 102, 176804 (2009).

[6] M. Katsnelson, K. Novoselov, and A. Geim, Nat. Phys. 2, 620 (2006).

[7] V. Lukose, R. Shankar, and G. Baskaran, Phys. Rev. Lett. 98, 116802 (2007).

[8] H. Wei, S.-P. Chao, and V. Aji, Phys. Rev. B. 86, 041403(R) (2012).

[9] For a review, see M. O. Goerbig, Rev. Mod. Phys. 83, 1193 (2011).

[10] S. Katayama, A. Kobayashi, and Y. Suzumura, J. Phys. Soc. Jpn. 75, 054705 (2006).

[11] A. Kobayashi, S. Katayama, Y. Suzumura, and H. Fukuyama, J. Phys. Soc. Jpn. 76, 034711 (2007).

[12] K. Kajita, T. Ojiro, H. Fujii, Y. Nishio, H. Kobayashi, A. Kobayashi, and R. Kato, J. Phys. Soc. Jpn. 61, 23 (1992); N. Tajima, M. Tamura, Y. Nishio, K. Kajita, and Y. Iye, ibid. 69, 543 (2000); N. Tajima, S. Sugawara, R. Kato, Y. Nishio, and K. Kajita, Phys. Rev. Lett. 102, 176403 (2009).

[13] N. Tajima, A. Ebina-Tajima, M. Tamura, Y. Nishio, and K. Kajita, J. Phys. Soc. Jpn. 71, 1832 (2002).

[14] K. Miyagawa, M. Hirayama, M. Tamura, and K. Kanoda, J. Phys. Soc. Jpn. 79, 063703 (2010).
[15] N. Tajima, A. Tajima, M. Tamura, R. Kato, Y. Nishio, and K. Kajita, J. Phys. IV (France) 114, 263 (2004).

[16] T. Choji, A. Kobayashi, and Y. Suzumura, J. Phys. Soc. Jpn. 80, 074712 (2011).

[17] M. O. Goerbig, J.-N. Fuchs, G. Montambaux, and F. Piéchon, Europhys. Lett. 85, 57005 (2009).

[18] C. L. Lu, C. P. Chang, Y. C. Huang, R. B. Chen, and M. L. Lin, Phys. Rev. B 73, 144427 (2006); J. Nilsson, A. H. Castro Neto, N. M. R. Peres, and F. Guinea, ibid. 73, 214418 (2006); M. Koshino and T. Ando, ibid. 73, 245403 (2006); F. Guinea, A. H. Castro Neto, N. M. R. Peres, ibid. 73, 245426 (2006); B. Partoens and F. M. Peeters, ibid. 74, 075404 (2006); S. Latil and L. Henrard, Phys. Rev. Lett. 97, 036803 (2006); V. P. Gusynin, S. G. Sharapov, and J. P. Carbotte, Phys. Rev. B 75, 165407 (2007).

[19] N. M. R. Peres and E. V. Castro, J. Phys.: Condens. Matter 19, 406231 (2007).

[20] M. O. Goerbig, J.-N. Fuchs, G. Montambaux, and F. Piéchon, Phys. Rev. B 78, 045415 (2008).

[21] T. Morinari, T. Himura, and T. Tohyama, J. Phys. Soc. Jpn. 78, 023704 (2009).

[22] D. B. Chklovskii, B. I. Shklovskii, and L. I. Glazman, Phys. Rev. B 46, 4026 (1992).

[23] K. Lier and R. R. Gerhardts, Phys. Rev. B 50, 7757 (1994).

[24] The study of this effect is delegated to future work.

[25] M. Assili, S. Haddad, and W. Kang, Phys. Rev. B 91, 115422 (2015).

[26] N. Zettili, Quantum Mechanics: Concepts and Applications, 2nd ed. (Wiley, 2009).

[27] J. Sári, C. Tőke and M. O. Goerbig, Phys. Rev. B 90, 155446 (2014).

[28] R. R. Nair, P. Blake, A. N. Grigorenko, K. S. Novoselov, T. J. Booth, T. Stauber, N. M. R. Peres, and A. K. Geim, Science 320, 1308 (2008). 